\title{
Resource Management in Multi-hop Mobile Small Cell Networks
}

\author{
Ade Syaheda Wani Marzuki, Iftekhar Ahmad, Daryoush Habibi and Quoc Viet Phung \\ Centre for Communications and Electronics Research, Edith Cowan University, Australia \\ Email: amarzuki@our.ecu.edu.au
}

\begin{abstract}
The deployment of mobile small cells has been identified as an effective strategy in delivering high data rates and providing seamless connectivity to a group of vehicular users. Although this approach can facilitate high data rates and ubiquitous wireless access, it introduces a new set of challenges, for instance, frequent changes in the interference set, requirements for more spectrum, high energy consumption and frequent handover. In this paper, we present a multi-hop mobile small cell network ( $\mathrm{SCN}$ ) that can facilitate wireless access to mobile nodes that do have direct transmission links to small cell base stations. We then formulate a solution to the frequency allocation problem for a multi-hop mobile SCN based on an optimization model. Considering the complexity of the optimization solution, we present a backhaul-aware frequency allocation solution based on the time-varying graph coloring concept. Simulation results confirm that the proposed solution outperforms an existing greedy solution by a significant margin in terms of throughput, spectral efficiency and fairness index.
\end{abstract}

Keywords-Mobile small cells, backhaul resource management, multi-hop transmission.

\section{INTRODUCTION}

Recent advances in mobile and wireless communication technologies have created unprecedented challenges for network operators in providing high data rates to end-users. Global mobile traffic is predicted to reach 35 exabytes per month by 2020 [1], and to accommodate this exponential growth of mobile data traffic, the fifth generation (5G) of communication systems is expected to introduce new innovative strategies. One such strategy is the widespread deployment of small cells. These small cells allow frequency reuse in neighboring small cells, thus increasing the network spectral efficiency [2-4]. Moreover, the transmission distance between receivers and transmitters can be reduced in a small cell, which would also contribute to lower energy consumption.

The deployment of small cells is not limited to fixed cells. Small cells can be deployed on moving nodes, for example, vehicles, to facilitate higher data rates to users in vehicular environments [3]. In mobile SCNs, a small vehicle (e.g., car or bus) can be modeled as a small cell, while a large vehicle, (e.g., train) can incorporate multiple small cells. The small cell access points are installed inside the vehicle, thus eliminating vehicle wall penetration losses. Therefore, a better signal quality is achieved. Various research efforts have been made to enable mobile small cell deployments in $5 \mathrm{G}$ networks. In [4-7], small cells are used as mobile relays in an existing macrocellular network to improve the network performance and extend coverage in hot-spots or cell-edge areas. In their work, these small cells backhaul their user traffic via macrocell base stations.

While the deployment of mobile small cells can enhance user Quality of Service (QoS) in vehicular environments, this introduces another dimension into the mobility management challenge, for instance, interference mitigation, bandwidth management, and wireless backhaul architecture. Resource allocation is an important issue that must be considered due to the dynamic change in topology of mobile small cells. This dynamic topology causes the interference set for each small cell to change frequently; thus the existing resource allocation schemes proposed for fixed small cells are not suitable for mobile small cells.

The resource allocation issues for mobile small cells were discussed in [4, 8-9]. In [4], the spectral and energy efficiency of a mobile femtocell network, for various resource partitioning schemes were discussed. Their results show that mobile femtocells can increase the network spectral efficiency and QoS. A cluster-based resource allocation scheme for deterministic mobile femtocells was proposed in [8]. The authors then extended their work by formulating an iterative resource allocation algorithm [9] to solve joint resource allocation problem in mobile femtocell networks. Mobile small cells can move at vehicular speeds and the maximum speed can reach up to $360 \mathrm{~km} / \mathrm{h}$ for some nodes (e.g., bullet trains) $[10,11]$. Thus, handover is another major challenge for mobile SCNs. In [6], it was shown that network providers would face no major handover and resource allocation issue if the mobile small cells would move at low speeds. However, if these cells move at high speeds, their QoS starts to degrade due to the limitations of existing resource management schemes. In order to address this issue, in our previous work [12], we presented a time-varying resource allocation solution for mobile small cells with deterministic mobility (e.g., public transport). In this work, we extended our research into a multihop mobile SCN and developed a resource management scheme for multi-hop mobile SCNs which can accommodate nodes with varying mobility pattern (e.g., car, van, private bus, ambulance). In this paper, we first present a multi-hop mobile $\mathrm{SCN}$ and establish the resource management challenge in the context of a multi-hop mobile SCN. We then present an optimization model to address the challenge. Considering the complexity of the optimization approach and the need for a real-time solution, we present a resource management scheme based on the time-varying graph coloring approach. In Section II of this paper, we explain the approach of our solution. In Section III, we present the analytical model and formulate the solution as an optimization model. Section IV presents a real- 